\title{
A spectroscopic survey of the WNL population in the LMC: very preliminary results
}

Olivier Schnurr, Anthony F.J. Moffat, Nicole St-Louis, Cedric Foellmi, and Gwen Skalkowski

Département de Physique, Université de Montréal, C.P.6128, Succ. Centre-ville, Montréal, QC H3C3J7, Canada

Michael M. Shara

Department of Astrophysics, American Museum of Natural History, Central Park West at 79th Street, New York, NY 10024-5192, USA

Virpi S. Niemela

Facultad de Ciencias Astronómicas y Geofísicas, Universidad Nacional de La Plata, Paseo del Bosque s/n, B1900FWA, La Plata, Argentina

\author{
Stéphane Vennes \\ Department of Mathematics, Australian National University, \\ Canberra, ACT 0200, Australia
}

Abstract. We present very preliminary results of a spectroscopic survey of all known 47 WNL stars in the LMC.

\section{Introduction}

The LMC is a perfect laboratory to study the effects of the metallicity $Z$ on the evolution of massive stars: (a) reddening is small and almost uniform; $(b)$ distances are well known; $(c) Z$ is lower than in our Galaxy $\left(Z_{\mathrm{LMC}}=0.3 \mathrm{Z}_{\mathrm{MW}}\right)$ and quite uniform; and $(d)$ the sample of WR stars is complete and large enough to obtain statistically significant results.

We are currently undertaking a large spectroscopic survey of all 47 known WNL stars in the LMC. In a first campaign, we have been awarded 49 nights at several 2m-class telescopes (CASLEO, MSO, SAAO, and CTIO) in order to obtain low-resolution $\left(2.3 \AA \mathrm{pix}^{-1}\right)$ spectra of the $41 \mathrm{WNL}$ stars accessible from the ground (for the seven remaining stars, HST is to be used). The goals of this study are:

(i) to determine the binary frequency among the WNL population of the LMC. Is binarity a major channel for WR formation in low- $Z$ environments, following the scenarios of Vanbeveren, van Rensbergen \& de Loore (1998)? Is there a dichotomy of the binary frequency among WN8,9 and WN6,7 stars, as has been 

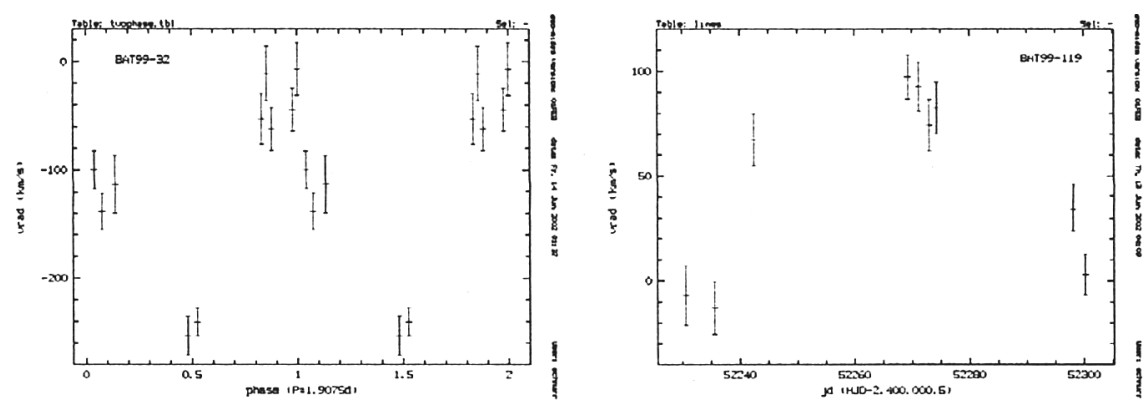

Figure 1. Left: BAT99-32 (WN6h) is a known binary with a period of $1.9075 \mathrm{~d}$ (M89). Graph shows our RVs plotted into corresponding phase. We can confirm the binary nature of this star. Right: BAT99-119 (WN6h) is a suspected binary with a tentative period of $25.17 \mathrm{~d}$ (M89). Our RVs confirm significant variability, although the period seems to be considerably longer, if this star is indeed a binary. More data are required.

reported by Moffat (1989, hereafter M89)?

(ii) to determine the masses of the WNL stars. Extremely luminous stars of the hydrogen-rich subgroup (denoted WNLh) are supposedly MS or early post-MS objects, thus still core hydrogen burning. They hence have the potential to be the most massive stars observable;

(iii) to study the wind-wind collision (WWC) effects in identified binaries. Modeling the excess emission due to WWC is a powerful tool to derive the orbital inclination angle, needed to obtain absolute masses (Lührs 1997; Hill, Moffat \& St-Louis 2001); and

(iv) to study line profile variations in identified single stars in order to search for large-scale structures in the WR wind, like co-rotating interacting regions (CIR).

\section{Results}

Very preliminary results of radial velocity (RV) analyses for selected stars are given (see Figure 1). RVs were measured by cross-correlation of the He II $\lambda 4686$ emission, using the best spectrum as template.

\section{References}

Hill, G., Moffat, A.F.J., St-Louis, N. 2001, MNRAS 318, 402

Lührs, S. 1997, PASP 109, 504

Moffat, A.F.J. 1989, ApJ 347, 127

Vanbeveren, D., van Rensbergen, W., de Loore, C. 1998, The Brightest Binaries (Dordrecht: Kluwer). 